\title{
HOW WE CAN MAKE SENSE OF CONTROL-BASED INTUITIONS FOR LIMITED ACCESS CONCEPTIONS OF THE RIGHT TO PRIVACY
}

\author{
Björn Lundgren
}

$\prod$

HERE IS a long-standing discussion on whether privacy and/or the right to privacy should be conceptualized in terms of limited access or control trol-based conceptions are among the most popular conceptions of privacy and the right to privacy, such conceptions suffer from various counterexamples. ${ }^{2}$ However, those who argue against control-based conceptions of privacy or the right to privacy rarely attempt to explain how competing conceptions of privacy or the right to privacy can make sense of some arguably strong control-based intuitions. For example, while presenting a dilemma against control-based conceptions of privacy, I myself have acknowledged that there are strong intuitions in favor of control-based conceptions of privacy, noting-somewhat summarily - that perhaps these intuitions can be better explained by contextual accounts. ${ }^{3}$ This is potentially problematic because, even if we find the counterexamples convincing, we may similarly find the control-based intuitions to be strong. Moreover, many recent counterexamples are only concerned with control-based conceptions of privacy, not the right to privacy. Indeed, the dilemma I present is only a dilemma for control-based conceptions of privacy. However, given that privacy is the object of the right to privacy, it follows that if privacy

1 I introduced the term "private matters" in "A Dilemma for Privacy as Control." This term refers to the things we should have control over or things that others should have limited access to (e.g., personal information and our bodies).

2 See, e.g., Parent, "Privacy, Morality, and the Law," "Recent Work on the Concept of Privacy," and "A New Definition of Privacy for the Law"; Macnish, "Government Surveillance and Privacy in a Post-Snowden World"; Lundgren, "A Dilemma for Privacy as Control"; and Solove, Understanding Privacy.

3 Lundgren, "A Dilemma for Privacy as Control," 165-66n1. 
cannot be defined in terms of control, neither should the right to privacy. ${ }^{4}$ This raises the question of whether conceptual consistency is more important than intuitions in determining the right way to conceptualize the right to privacy.

In this article, I aim to remedy this situation by showing how limited access conceptions of the right to privacy can satisfy control-based intuitions while providing a satisfactory alternative explanation for these intuitions. The focus is only on the right to privacy, not privacy as such. ${ }^{5}$ Furthermore, the focus is only on the moral right to privacy, not the legal right to privacy. Moreover, my intention is not to defend control-based intuitions; rather, I will present and explain these intuitions and then show how a limited access conception can be modified to address these intuitions and yield the same conclusion as a control-based conception. The question of whether we should make such a modification is not something I aim to settle in this paper, although I will make brief comments on this subject.

The modifications I will propose herein are based on the idea that risk taking can violate or infringe upon the right to privacy. I proposed this idea previously, based on Sven Ove Hansson's more general idea of a pro tanto right against risks. ${ }^{6} \mathrm{I}$ also defended this idea more explicitly in a recent publication, in which I argued that it makes sense to think of the right to privacy as being violated or infringed upon in cases where someone attempts to access private matters. Moreover, I qualified these attempts in terms of substantial risks. ${ }^{7}$ The publication also critiqued an analysis of the right to privacy in terms of negative control by Jakob Thraine Mainz and Rasmus Uhrenfeldt. ${ }^{8}$ I argued that my presumptions followed from Mainz and Uhrenfeldt's arguments, and these presumptions can

4 Lundgren, “A Dilemma for Privacy as Control," 166.

5 There are at least three reasons for this. First, as I noted in "A Dilemma for Privacy as Control," control-based conceptions are mostly popular due to control-based conceptions of the right to privacy, not due to control-based conceptions of privacy. Second, intuitions in favor of control-based conceptions are arguably stronger for conceptions of the right to privacy, rather than conceptions of privacy as such. Third, as noted above, some substantial arguments against control-based accounts of privacy deal primarily with privacy rather than the right to privacy.

6 Lundgren, "Against AI-Improved Personal Memory"; Hansson, "Ethical Criteria of Risk Acceptance." Hansson called this right prima facie, but as I have noted (Lundgren, "Against AI-Improved Personal Memory," 229n39), what he proposed is better described as a pro tanto right.

7 Lundgren, "Confusion and the Role of Intuitions in the Debate on the Conception of the Right to Privacy."

8 Mainz and Uhrenfeldt, “Too Much Info.” 
also be used to create a counterexample against their proposed definition of the right to privacy. ${ }^{9}$

Lauritz Aastrup Munchalso proposed the idea that the right to privacy should provide moral protection against risks. ${ }^{10}$ Munch's work focuses on the abuse of information. Moreover, similar to myself, he proposed this idea based on works concerning the normative aspects of risks, albeit by a different author (John Oberdiek). More recently, Munch provided an extensive defense of the idea that probabilistic inferences can violate the right to privacy. ${ }^{11}$

The aim of the present article differs from the aims of these prior works. More specifically, my aim is to show how control-based intuitions can be explained based on the idea that the right to privacy protects against certain forms of risk.

To simplify this discussion and show how these ideas can be generalized, I will introduce a simplified schema of necessary and jointly sufficient conditions for when a standard control-based conception $(C)$ and a standard, limited access-based conception $(L)$ are infringed upon or violated: ${ }^{12}$

C: A control (access) conception of the right to privacy implies that an individual A's right to privacy is infringed upon or violated if and only if another individual controls (access to) part of $A$ 's private matters.

$L$ : A limited access conception of the right to privacy implies that an individual A's right to privacy is infringed upon or violated if and only if another individual accesses part of $A$ 's private matters.

Note that $C$ only covers certain control-based conceptions of the right to privacy; later (in section II), I will turn to alternatives. I will begin section I by discussing

9 It should be noted that an unfortunate formulation in Lundgren, "Confusion and the Role of Intuitions in the Debate on the Conception of the Right to Privacy," may make it appear as if that article was the first source of this idea, ignoring both Lundgren, "Against AI-Improved Personal Memory"; and Munch, "The Right to Privacy, Control Over Self-Presentation, and Subsequent Harm." What I should have said was that the idea was relatively new and perhaps reasonably unknown to Mainz and Uhrenfeldt.

Munch, "The Right to Privacy, Control Over Self-Presentation, and Subsequent Harm."

Munch, "Privacy Rights and 'Naked' Statistical Evidence"; I will not discuss this article, as it was published after the present article was accepted.

By merely providing a schema, I am setting aside many issues that a complete conception of the right to privacy must consider (e.g., the role of informed consent, right forfeiture, and conditions - if any-when the right is overridden). What I am interested in here is whether we can modify the limited access conception $(L)$ to yield the same conclusion as control-based conceptions of the right to privacy in a situation where control-based intuitions appear to be very strong. 
one example of control-based intuitions from my previous writings. ${ }^{13}$ The aim of this example is to show how we can modify $L$ to yield the same evaluation as an application of $C$ while ensuring that the modification of $L$ does not turn into a control-based conception of the right to privacy. In section II, I will modify this example to consider alternative formulations of control-based conceptions of the right to privacy and to show how $L$ can be further modified. These examples are intended to show that generalizability of the idea that a limited access account of the right to privacy can be modified to explain control-based intuitions. Finally, in the last section, I will conclude and summarize.

In recent work, I mentioned an example in which a prisoner is held in a cell with a hatch that someone else controls (henceforth, the "prison hatch example"). ${ }^{14}$ If we accept $C$ (and set aside the issue of whether the right to privacy might have been forfeited or overridden), we should conclude that the prisoner's right to privacy is infringed upon or violated because the hatch is controlled by someone else and it follows that someone else controls (access to) the prisoner's private matters. Furthermore, proponents of control-based conceptions of the right to privacy would hold that even if the hatch is never opened, it may seem as if the prisoner's right to privacy is infringed upon or violated because the presence of the hatch affects the prisoner's control over (access to) their private matters. In contrast, if we accept $L$, we must recognize that if the hatch is not opened, then there is no infringement or violation of the right to privacy (i.e., the right to privacy would only be infringed upon in situations where the hatch is actually opened, allowing access to the prisoner's private matters). Proponents of limited access conceptions may argue that although the presence of the hatch may have negative effects on the prisoner, such as changes in their behavior (due to the potential of being surveilled), these effects can fully be explained by how it affects their autonomy, rather than their privacy. Nevertheless, this idea could also be used to speak in favor of control-based conceptions because of the close link between privacy and autonomy. Hence, I will show how it is possible to modify limited access conceptions to yield the same conclusion as control-based conceptions while explaining control-based intuitions in a way that retains a limited access conception.

As I noted in the introduction, the modification I will focus on herein recognizes that the right to privacy includes a right not to have others put one's

13 Lundgren, "A Dilemma for Privacy as Control."

14 Lundgren, "A Dilemma for Privacy as Control." 
privacy at substantial risk. Hansson argued for a general right against risk exposure, which can be overridden only under certain circumstances, such that "this [risk] exposure is part of an equitable social system of risk-taking that works to her advantage." ${ }^{15}$

The idea that the right to privacy should include protection against risk impositions can be defended in a variety of ways. Consider a situation in which Jane voluntarily performs an action @, knowing that @ has a high probability (e.g., > 99\%) of exposing a very private matter of Joe's. If we grant that exposing this very private matter would violate or infringe upon Joe's right to privacy, then should we not also grant that it would violate or infringe upon his right even if the risk is not actualized? I believe that the answer is yes, although it is important to note that the specific probability of the actualization of the risk may matter in this scenario, and I will consider this in my modification of $L$.

To more clearly understand the idea that the right to privacy should morally protect you against certain risks to your privacy, it may be useful to consider a practical example. Suppose, for example, that you store very personal information on a secure cloud service. Furthermore, suppose that someone hacks the security protection of this information so that it is accessible by anyone. Even if no one actually accesses this information, it would be reasonable to hold that the hacker has infringed upon or violated your right to privacy. Although one could alternatively argue that the hackers infringed upon or violated rights other than privacy, it would then be difficult to explain the difference between making privacy-sensitive information accessible and making privacy-insensitive information accessible. More importantly, the goal here is not to defend control-based intuitions but to show how a limited access conception can provide alternative explanations for control-based intuitions and reach the same result. While proponents of control-based conceptions would argue that risk impositions affect the right-bearer's control over their private matters, an alternative explanation is that an action that puts access under risk is an action that risks delimiting access and, hence, is an action that infringes or violates upon the right to limited access to one's private matters. ${ }^{16}$ If we accept this idea, then we can grant that proponents of $C$ are correct to consider the hatch as an infringement or violation of the

\section{Hansson, "Ethical Criteria of Risk Acceptance," 305, bracketed text added.}

Of course, control and risk taking can come apart, but this does not mean that control-based intuitions speak against intuitions in favor of the idea that a right to privacy should protect against privacy risks. In fact, as I have argued before, intuitions about risks to or attempted access may sometimes be in a better position to explain what are commonly taken to be intuitions in favor of control; see Lundgren, "Confusion and the Role of Intuitions in the Debate on the Conception of the Right to Privacy." 
right to privacy but wrong in their explanation for why the hatch is an infringement or violation. We can easily modify $L$ to satisfy this alternative explanation:

L-risk: A limited access conception of the right to privacy implies that an individual $A$ 's right to privacy is infringed upon or violated if and only if another individual $B$ either (1) accesses part of $A$ 's private matters $p$ or (2) makes it so that $p$ is at risk of being accessed by some individual $C$ (such that that $C$ is not $A$ ).

This would resolve the prison hatch example because the hatch puts A's private matters at risk. Risk exposure would also provide an alternative explanation to control-based intuitions while remaining congruent with the lack of control.

However, a few possible complications may arise with this conception. First, the meaning of " $B$ makes it so that" is not entirely clear. Setting aside complications about how to understand the concept of causation, there is a question as to who creates this risk. For example, is it the door maker; the state, which put $A$ in this situation; or the prison ward, who makes decisions about the whether to open the hatch? However, given that a similar problem arises with control-based conceptions, we can set this issue aside (given the limited purpose of this paper). Second, $A$ 's private matters are always at risk in a strict sense, making L-risk somewhat vague. What does it mean for $B$ to make it so that $A$ 's private matters are at risk if these matters are already at risk? We can solve this quite simply by saying, " $B$ makes it so that $\mathrm{p}$ is at (greater) risk," thus relativizing the proposal. However, this may raise another problem, as $B$ may be performing an action that only slightly increases the level of risk. That is, we may unintentionally say that one can violate or infringe upon another's right to privacy by simply performing an action that indirectly increases the level of risk to the other person's privacy by a mere fraction of a percentage. While some would agree that this is the correct understanding, we could also resolve this by requiring a substantial increase in risk. Alternatively, we could note that the privacy right against risk exposure is overridable, perhaps by modifying it according to Hansson's criteria for situations in which the right against risk exposure is overridden. ${ }^{17}$

In the previous section, I argued that, if we accept $C$, we recognize that the presence of the prison hatch infringes upon or violates the prisoner's privacy, even if it is never opened. Similarly, I showed how a limited access conception can be modified to reach the same conclusion. However, what if the hatch is not

17 Hansson, "Ethical Criteria of Risk Acceptance." 
only never opened but — unbeknownst to the prisoner-cannot be opened? To understand the intuition around this case it may be more illustrative to consider a dummy camera rather than a dummy prison hatch (henceforth, the "dummy prison hatch example"). ${ }^{18}$

Note that, according to $C$, the dummy prison hatch would not infringe upon or violate the prisoner's right to privacy. However, some proponents of control-based conceptions of the right to privacy may claim that what matters is not only control over private matters but that this control is a form of the individual's self-control. Although it may, again, be argued that such ideas conflate privacy with autonomy, I will nevertheless consider this claim herein. ${ }^{19}$

C-self: A control (access) conception of the right to privacy implies that an individual $A$ 's right to privacy is infringed upon or violated if and only if $A$ 's self-control over (access to) part of A's private matters is reduced.

According to $C$-self, the dummy prison hatch infringes upon or violates the prisoner's privacy because it affects the prisoner's self-control over their private matters. To see this spelled out in greater detail, consider Andrei Marmor's idea that the right to privacy is grounded in an interest to control how we present ourselves to others. ${ }^{20}$ While we may be skeptical of spelling out the right to privacy in terms of this interest, this idea can be used to illustrate how the dummy prison hatch may infringe upon the right to privacy. ${ }^{21}$ Simply put, the presence of a dummy hatch (or dummy camera) can affect how a person behaves. However, such an effect is explainable only if the presence of the hatch affects the person's beliefs or knowledge of potential surveillance by others. Thus, let us consider how $L$-risk can be further modified to address such intuitions.

Based on the arguments just considered, I will introduce a distinction be-

The comparison to the previous example would still hold. If we modify the previous example, the camera surveillance footage is not watched but nevertheless implies the substantial risk that it could be viewed. In the current example, a mounted dummy camera affects people's behavior because they do not know that the camera is a dummy.

9 Below, I will exemplify this view with ideas from Marmor, "What Is the Right to Privacy?" However, this idea has recently been defended in analyses of privacy (rather than the right to privacy) by Menges, "A Defense of Privacy as Control" and "Three Control Views on Privacy." As Menges notes (in "Three Control Views on Privacy") his arguments can be spelled out in defense of a conception of the right to privacy (as he plans to do). Moreover, Menges explains how his analysis of privacy differs from an analysis of autonomy ("A Defense of Privacy as Control"). Marmor, "What Is the Right to Privacy?"

\footnotetext{
For counterexamples, see Lundgren, "A Dilemma for Privacy as Control," 172n17. For a detailed critique, see Munch, "The Right to Privacy, Control Over Self-Presentation, and Subsequent Harm."
} 
tween belief in an actual possibility (risk) and an agent's epistemic or doxastic uncertainty as to whether something is at risk. To modify $L$-risk, we have two alternatives. First, we may consider A's privacy to be infringed upon or violated when $B$ makes $A$ believe that their private matters are at risk of being accessed by some individual $C$ (such that $C$ is not $A$ ). Second, we may consider A's privacy to be infringed upon or violated when $B$ makes $A$ uncertain as to whether their private matters are at risk of being accessed by some individual $C$ (such that $C$ is not $A$ ). We could also further qualify these alternatives (e.g., by requiring the degree of uncertainty to be substantial). Adding both conditions would produce the following:

L-risk and epistemic and doxastic uncertainty: A limited access conception of the right to privacy implies that an individual A's right to privacy is infringed upon or violated if and only if another individual $B$ either (1) accesses part of $A$ 's private matters, (2) makes it so that $p$ is at risk of being accessed by some individual $C$ (such that $C$ is not $A$ ), (3) makes it so that $A$ believes that their private matters are at risk of being accessed by some individual $C$ (such that $C$ is not $A$ ), or (4) makes it so that $A$ is (substantially) uncertain as to whether their private matters are at risk of being accessed by some individual $C$ (such that $C$ is not $A$ ).

Modifying $L$-risk accordingly would resolve the dummy prison hatch example, either because $A$ believes that the hatch can be opened and thus poses a risk or because $A$ is uncertain as to whether the hatch poses a risk. Arguably, if $A$ 's self-control (over how she presents herself) is affected, it is affected because $A$ does not know whether the hatch may be used (and similarly in the case of a dummy camera). That is, if we accept the basic intuitions here, then the right to privacy protects against manipulation of an agent's belief in (or knowledge of) the risk that others may access their private matters.

Keep in mind that the goal here is not to defend the conclusions about privacy rights infringements and violations that follow from accepting $C$-self or $L$-risk and epistemic and doxastic uncertainty. While I believe that such a conception of the right to privacy would conflate the right to privacy with the right to know that one's privacy is retained or protected, that discussion is beyond the aim and scope of this paper. The goal of this paper was merely to show that it is possible to provide an alternative explanation for control-based intuitions and a limited access conception of the right to privacy that satisfies these intuitions. By considering these alternatives, I hope to have shown that it is very likely that these type of modifications can be generalized. 


\section{CONCLUSIONS}

Above, I have shown how limited access conceptions of the right to privacy can provide alternative explanations to control-based intuitions about the right to privacy. Indeed, we can modify a limited access conception of the right to privacy to yield the same conclusions as variants of control-based conceptions of the right to privacy. First, we saw how one can make sense of some very common control-based intuitions by adapting the idea that the right to privacy (in terms of limited access) also includes a right against substantial risk impositions to one's privacy (or private matters). Next, we turned to conceptions of the right to privacy that suppose a closer link between privacy and autonomy. I showed how the idea that we can infringe upon or violate someone's right to privacy by affecting their self-control can be addressed by using a limited access conception of the right to privacy and introducing criteria related to the agent's knowledge or beliefs. Specifically, an agent's right to privacy can be infringed upon or violated if their beliefs (or knowledge) about access to their private matters are (substantially) affected. The question of whether we should accept these revisions is partly beyond the scope of this paper, but I hope these examples can show how this defense of limited access analyses of the right to privacy can be generalized and adapted to address more specific control-based intuitions (as I did in previous writings). ${ }^{22}$

Umeå University, Institute for Futures Studies, and Stockholm University bjorn.lundgren@umu.se

\section{REFERENCES}

Hansson, Sven Ove. "Ethical Criteria of Risk Acceptance." Erkenntnis 59, no. 3 (November 2003): 291-309.

Lundgren, Björn. "Against AI-Improved Personal Memory." In Aging Between Participation and Simulation: Ethical Dimensions of Socially Assistive Technologies in Elderly Care, edited by Jochen Vollmann, Johanna Hovemann, and Joschka Haltaufderheide, 223-34. Berlin: De Gruyter, 2020.

22 Lundgren, "Confusion and the Role of Intuitions in the Debate on the Conception of the Right to Privacy." Lastly, I want to thank an anonymous reviewer for Journal of Ethics and Social Philosophy. Moreover, I want to acknowledge that this work was supported by the Wallenberg AI, Autonomous Systems and Software Program-Humanities and Society (WASP-HS), funded by the Marianne and Marcus Wallenberg Foundation and the Marcus and Amalia Wallenberg Foundation (grant number: MMW 2018.0116). 
. "Confusion and the Role of Intuitions in the Debate on the Conception of the Right to Privacy." Res Publica (2021). https://doi.org/10.1007/s11158 -020-09495-9.

—. "A Dilemma for Privacy as Control." Journal of Ethics 24, no. 2 (June 2020): $165-75$.

Macnish, Kevin. "Government Surveillance and Privacy in a Post-Snowden World." Journal of Applied Philosophy 35, no. 2 (May 2018): 417-32.

Mainz, Jakob Thrane, and Rasmus Uhrenfeldt. "Too Much Info: Data Surveillance and Reasons to Favor the Control Account of the Right to Privacy." Res Publica 27, no. 2 (May 2021): 287-302.

Marmor, Andrei. "What Is the Right to Privacy?" Philosophy and Public Affairs 43, no. 1 (Winter 2015): 3-26.

Menges, Leonhard. "A Defense of Privacy as Control." Journal of Ethics 25, no. 3 (September 2021): 385-402.

- "Three Control Views on Privacy." Social Theory and Practice (forthcoming).

Munch, Lauritz Aastrup. "Privacy Rights and 'Naked' Statistical Evidence." Philosophical Studies 178, no. 1 (November 2011): 3777-95.

- "The Right to Privacy, Control over Self-Presentation, and Subsequent Harm." Journal of Applied Philosophy 37, no. 1 (February 2020): 141-54.

Parent, W.A. "A New Definition of Privacy for the Law." Law and Philosophy 2, no. 3 (December 1983): 305-38.

- "Privacy, Morality, and the Law." Philosophy and Public Affairs 12, no. 4 (Autumn 1983): 269-88.

- "Recent Work on the Concept of Privacy." American Philosophical Quarterly 20, no. 4 (October 1983): 341-55.

Solove, Daniel J. Understanding Privacy. Cambridge, MA: Harvard University Press, 2008. 